\title{
On Some Aspects of Strong Risk Class and Associated Ordering
}

\author{
Mervat Mahdy* \\ 2018 \\ Received 24 March 2016 \\ Accepted 19 November 2017
}

\begin{abstract}
The paper opens up a new aging class and a new stochastic orders which is depend on risk class, that plays vital role in the reliability theory, finance topics, stochastic orders, and the economic theory. The article presents some new interesting implications and characterizations concerning this class. In addition, we list a series of inequalities that provide bounds for strong risk and some aging classes. Furthermore, a sufficient condition for a probability distribution to have a new class is provided. In addition, The paper demonstrates the preservation properties of a new stochastic order under some reliability operations such as mixture, and convolution. Moreover, some new reliability concepts based on discrete lifetime random variable are studied.

Keywords: Stochastic orders; Mean inactivity time class; Strong mean inactivity time class; Variance inactivity time; Preservation; Risk measure.

2000 Mathematics Subject Classification: 60E15, 90B25, 91B30
\end{abstract}

\section{Introduction and Motivations}

Consider a probability density function $f(t)$ for a lifetime random variable $X$ with distribution function $F(t)$ and survival function $\bar{F}(t)=1-F(t), t \in$ $\mathbb{R}^{+}$. In addition, we assume that the mean life $\mu_{X}=\int_{0}^{\infty} \bar{F}(u) d u$ and the variance $\sigma_{X}^{2}$ are finite. Likewise, let $Y$ be the second lifetime random variable with the density function $g(t)$, distribution function $G(t)$ and survival function $\bar{G}(t)=1-G(t) ; t \in \mathbb{R}^{+}$. Furthermore, the mean life $\mu_{Y}=\int_{0}^{\infty} \bar{G}(u) d u$ and variance $\sigma_{Y}^{2}$ are both assumed to be finite. Let $l_{X}=\inf \left\{t \in \mathbb{R}^{+}: F(t)>0\right\}$, $u_{X}=\sup \left\{t \in \mathbb{R}^{+}: F(t)<1\right\}, \Omega_{X}=\left(l_{X}, u_{X}\right), l_{Y}=\inf \left\{t \in \mathbb{R}^{+}: G(t)>0\right\}$, $u_{Y}=\sup \left\{t \in \mathbb{R}^{+}: G(t)<1\right\}$ and $\Omega_{Y}=\left(l_{Y}, u_{Y}\right)$. Let $X$ has reversed hazard rate function $\widetilde{r}_{F}(t)=f(t) / F(t), t>l_{X}$ and $Y$ has reversed hazard rate function

${ }^{*}$ Corresponding author: Dept. of Statistics, Mathematics and Insurance, College of Commerce, Benha University, Egypt. Email. drmervat.mahdy@fcom.bu.edu.eg. Tel: +20-122068-2460, fax: +20-13-323-0860. 
$\widetilde{r}_{G}(t)=g(t) / G(t), t>l_{Y}$. Then, the mean inactivity lifetime functions, and variance inactivity lifetime functions respectively are defined by

$$
\begin{gathered}
m_{F}(t)=\left\{\begin{array}{cl}
\frac{1}{F(t)} \int_{0}^{t} F(u) d u, & \text { if } t>l_{X} \\
0, & \text { if } t \leq l_{X}
\end{array}\right. \\
m_{G}(t)=\left\{\begin{array}{cl}
\frac{1}{G(t)} \int_{0}^{t} G(v) d v, & \text { if } t>l_{Y} \\
0, & \text { if } t \leq l_{Y}
\end{array}\right. \\
\sigma_{F_{(t)}^{2}}^{2}(t)=\left\{\begin{array}{cl}
\frac{2 \int_{0}^{t} \int_{0}^{y} F(u) d u d y}{F(t)}-m_{F}^{2}(t), & \text { if } t>l_{X} \\
0, & \text { if } t \leq l_{X}
\end{array}\right.
\end{gathered}
$$

and

$$
\sigma_{G_{(t)}}^{2}(t)=\left\{\begin{array}{cl}
\frac{2 \int_{0}^{t} \int_{0}^{y} G(u) d u d y}{G(t)}-m_{G}^{2}(t), & \text { if } t>l_{Y} \\
0, & \text { if } t \leq l_{Y}
\end{array} .\right.
$$

The following definitions are essential for this study.

Definition 1.1. The distribution function $F($.$) of the random variable X$ is said to have the following characteristics:

(i) A decreasing reversed hazard rate $(D R H R)$, if $\widetilde{r}_{F}(t)$ is a decreasing function in $t$, or if $F(t)$, is logarithmically concave in $t \in R^{+}$.

(ii) An increasing strong mean past lifetime class if $\int_{0}^{t} x F(x) d x / F(t)$ is nondecreasing for all $t>l_{X}$.

The following stochastic orders are defined in Nanda et al. (2003); Shaked and Shanthikumar (2007); Mahdy (2012); Kayid and Izadkhah (2014):

Definition 1.2. Let $X_{1}$ and $X_{2}$ be two non-negative and absolutely continuous random variables, with the distribution functions $F_{1}($.$) and F_{2}($.$) , density$ functions $f_{1}($.$) and f_{2}($.$) , reversed hazard rate functions \widetilde{r}_{F_{1}}($.$) and \widetilde{r}_{F_{2}}($.$) ,$ mean past lifetime functions $m_{F_{1}}($.$) and m_{F_{2}}($.$) , and the variance past lifetime$ functions $\sigma_{F_{1(x)}}^{2}($.$) and \sigma_{F_{2(x)}}^{2}($.$) , respectively. Hence, X_{1}$ is smaller than or equal $X_{2}$ in the following cases:

(i) A reversed residual lifetime ordering $\left(X_{1} \leq_{R H R} X_{2}\right)$, if $\widetilde{r}_{F_{1}}\left(x_{2}\right) \leq \widetilde{r}_{F_{2}}\left(x_{2}\right)$, for all $x_{2}>0$ or $\left\{F_{1}\left(x_{1}\right) / F_{1}\left(x_{2}\right)\right\} \geq\left\{F_{2}\left(x_{1}\right) / F_{2}\left(x_{2}\right)\right\}$, for all $x_{1} \leq x_{2}$.

(ii) A mean past lifetime order $\left(X_{1} \leq_{M P} X_{2}\right)$, if $m_{F_{1}}\left(x_{2}\right) \geq m_{F_{2}}\left(x_{2}\right)$, for all $x_{2}>0$.

(iii) A variance past lifetime order $\left(X_{1} \leq_{V P} X_{2}\right)$ if $\sigma_{F_{1(x)}}^{2}\left(x_{2}\right) \geq \sigma_{F_{2(x)}}^{2}\left(x_{2}\right)$, for all $x_{2}>0$.

(iv) A strong mean past lifetime order $\left(X_{1} \leq_{S M P} X_{2}\right)$, if $\int_{0}^{x_{2}} x F_{1}(x) d x / F_{1}\left(x_{2}\right) \geq$ $\int_{0}^{x_{2}} x F_{2}(x) d x / F_{2}\left(x_{2}\right)$, for all $x_{2} \in \mathbb{R}^{+}$. 
(v) A likelihood ratio order $\left(X_{1} \leq_{L R} X_{2}\right)$, if $f_{1}(u) f_{2}(v) \geq f_{1}(v) f_{2}(u)$, for all $u \leq v$.

Recently, Mahdy (2012) shown that, if $X_{1} \leq_{V P} X_{2}$, we can be get that $X_{1}(\varphi) \leq_{V P} X_{2}(\varphi)$ for any concave transformation $\varphi$. In addition, $X_{1}(\varphi) \leq_{V P}$ $X_{2}(\varphi) \Rightarrow X_{1} \leq_{V P} X_{2}$ for any strictly increasing function $(\varphi)$.

In insurance, if we represent the distribution by the appropriate random variable $\mathbf{X}$, and let $\theta$ present the risk measure functional, then

$$
\theta: \mathbf{X} \rightarrow \mathbb{R} .
$$

Let an insurance contract in a specified period $(0, a)$ and let $\Omega$ be the state space. If none of the risks specified in the policy contract happen during the policy term, then the policy holder has no monetary compensation for the paid premiums. Then the loss that the difference between amount of compensation (that denoted by $\mathbb{C}$ ) and total losses resulting from achieve state $\alpha$ occurs (that denoted by $\varphi\left(X_{(\alpha)}\right)=X^{2}$ is convex function) is $\left[\mathbb{C}-\varphi\left(X_{(\alpha)}\right)\right.$ | $\left.X_{(\alpha)} \leq \mathbb{C}\right]$, where $X$ is premiums state $\alpha$. Suppose we associate a "risk" as $\operatorname{Var}\left[\mathbb{C}-\varphi\left(X_{(\alpha)}\right) \mid X_{(\alpha)} \leq \mathbb{C}\right]$, that is convex. We called it a strong risk function, $S R$, it is natural to demand that a risk function has this non-decreasing. Then the variance of random variable $\left[\mathbb{C}-\varphi\left(X_{(\alpha)}\right) \mid X_{(\alpha)} \leq \mathbb{C}\right]$ base on convex function, $\varphi\left(X_{(\alpha)}\right)$, can use both in studying the effects of investor and analyst beliefs on securities trading. Also, we can use $S R$ in measure of dispersion of returns of investment portfolio. In addition, $S R$ measures the variability of a security's returns relative to market index or a particular benchmark. If $\operatorname{Var}\left[\mathbb{C}-\varphi\left(X_{(\alpha)}\right) \mid X_{(\alpha)} \leq \mathbb{C}\right]$ is greater than that of the benchmark, then the financial instrument is thought to be more perilous than the benchmark price. Low $\operatorname{Var}\left[\mathbb{C}-\varphi\left(X_{(\alpha)}\right) \mid X_{(\alpha)} \leq \mathbb{C}\right]$ may mean lower risk.

As a result, the objective study is to achieve two aims. The first aim is consider a new nonparametric class of distributions, depended on $S R$ with introduce some characterizations, and some preservation. The second aim is suggest a new technique that improves the comparison between two distributions with some characterizations, preservation results, and applications for strong risk order. Section 2 provides some properties and application of new class. Furthermore, the behavior of $S R$ is presented. In addition, we listed a series of inequalities that provide bound for $S M P$ and $S R$ functions. Also, a sufficient conditions for a probability distribution to have a new class are studied. In Section 4, the relationships between the strong risk order and others stochastic orders will be discussed. Furthermore, useful properties and characterization of the strong risk order is studied. In addition, we establish closure properties of strong risk order under relevant reliability operations such as convolution, mixture and transformation. Finally, Section 5 discusses the useful applications in statistical reliability theory involving the strong risk order and the increasing strong risk class. 


\section{Characterizations of SR Class}

Let a random variable $X$ have the density function $f($.$) , distribution func-$ tion $F($.$) , and reversed hazard rate \widetilde{r}_{F}($.$) . Setting u(y)=\int_{0}^{y} F(x) d x$ and $E\left[U^{2} \mid \kappa\right]=\int_{0}^{\infty} u^{2} d F[u \mid \kappa] d \kappa$, and by using integration by parts one has $S R$ function we obtain:

$$
\theta_{F}(\kappa)=(\kappa(1-\kappa))^{2}+\frac{4 \int_{0}^{\kappa} x\left(\kappa-x^{2}\right) F(x) d x}{F(\kappa)}-S_{F}^{2}(\kappa) .
$$

where $S_{F}(\kappa)=\int_{0}^{\kappa} x F(x) d x / F(\kappa)$, it is called strong mean past lifetime (SMP). Then, after some algebraic calculations, we obtain

$$
\begin{aligned}
E\left[\left(\kappa-X^{2}\right)^{2} \mid X \leq \kappa\right] & =\theta_{F}(\kappa)+S_{F}^{2}(\kappa) \\
& =(\kappa(1-\kappa))^{2}+\frac{4\left[\kappa \int_{0}^{\kappa} \int_{0}^{x} F(u) d u d x-\int_{0}^{\kappa} x^{3} F(x) d x\right]}{F(\kappa)} .
\end{aligned}
$$

If we let $\pi_{F}^{i}(x)=4 \int_{0}^{x} y^{i} F(y) d y / F(x)$, then (2.1) is equivalent to

$$
\theta_{F}(\kappa)=(\kappa(1-\kappa))^{2}+S_{F}(\kappa)\left(2 \kappa-S_{F}(\kappa)\right)-\pi_{F}^{3}(\kappa) .
$$

In other words, $(2.2)$ is equivalent to

$$
\begin{aligned}
\theta_{F}(\kappa) & =\kappa^{2}(1-2 \kappa)+S_{F}(\kappa)\left(2 \kappa-S_{F}(\kappa)\right)+E\left(X^{4} \mid X \leq \kappa\right) / F(\kappa), \\
& =(\kappa(1-\kappa))^{2}+2 \kappa\left(\sigma_{F}^{2}(\kappa)+m_{F}^{2}(\kappa)\right)-4 \pi_{F}^{3}(\kappa)-S_{F}^{2}(\kappa) .
\end{aligned}
$$

In such cases we express $F \in \mathcal{L}$.

Definition 2.1. The non-negative random variable $X$ is said to be smaller than or equal to $Y$ in strong risk order $\left(X \leq_{\theta(\kappa)} Y\right)$ if

$$
\frac{\int_{0}^{\kappa} x\left(\kappa-x^{2}\right) F(x) d x}{\int_{0}^{\kappa} x\left(\kappa-x^{2}\right) G(x) d x}, \text { is increasing in } \kappa, \text { for all } \kappa \in \mathbb{R}^{+} .
$$

Suppose we refer to strong risk with the symbol $\mathbf{S}$, an increasing function with abbreviate $\mathbf{I}$ and for decreasing function with symbol $\mathbf{D}$, we can define the following two classes of lifetime:

(i) $\mathbf{S}_{\mathbf{D}}=\left(F \in \mathcal{L}: \theta_{F}(\kappa)\right.$, for all $\kappa$, is a $\left.\mathbf{D}\right)$,

(ii) $\mathbf{S}_{\mathbf{I}}=\left(F \in \mathcal{L}: \theta_{F}(\kappa)\right.$, for all $\kappa$, is a $\left.\mathbf{I}\right)$. 
Clearly $\mathbf{S}_{\mathbf{D}}$ and $\mathbf{S}_{\mathbf{I}}$ form a pair of dual classes base on the distinguishing $\theta_{F}(\kappa)$. In current section, we introduce and explain the properties and applications of the $\mathbf{S}_{\mathbf{D}}$ and $\mathbf{S}_{\mathbf{I}}$ classes. The sufficient conditions for $F($.$) to have the$ $\mathbf{S}_{\mathbf{D}}$ and $\mathbf{S}_{\mathbf{I}}$ are provided by next theorem.

Theorem 2.1: Let $T$ denote the lifetime of an equipment with $S_{F}(\kappa)$, $\pi_{F}^{3}(\kappa), \widetilde{r}_{F}(\kappa)$ and $S_{F}^{\searrow}(\kappa)=\partial S_{F}(\kappa) / \partial \kappa$. If

$$
S_{F}(\kappa)\left(1-S_{F}^{\searrow}(\kappa)\right)+\kappa S_{F}^{\searrow}(\kappa)>(\leq) \frac{-1}{2}\left(2 \kappa(1-3 \kappa)+\widetilde{r}_{F}(\kappa) \pi_{F}^{3}(\kappa)\right), \kappa>0,
$$

or

$\widetilde{r}_{F}(\kappa) \theta_{F}(\kappa)<(\geq) 2 \kappa(1-2 \kappa)+2(1-\kappa) S_{F}(\kappa)+\widetilde{r}_{F}(\kappa)\left[S_{F}^{2}(\kappa)+\kappa^{2}(1-\kappa)^{2}\right]$.

Then $T \in \mathbf{S}_{\mathbf{I}}\left(\mathbf{S}_{\mathbf{D}}\right)$.

Proof. The first differentiation of $S_{F}(\kappa)$ can be rewritten as

$$
\begin{aligned}
S_{F}^{\searrow}(\kappa) & =\frac{\partial}{\partial \kappa} u(\kappa) / F(\kappa)=\kappa-\frac{f(\kappa) u(\kappa)}{F^{2}(\kappa)}, \\
& =1-\widetilde{r}_{F}(\kappa) S_{F}(\kappa) .
\end{aligned}
$$

By differentiating (2.2) with respect to $\kappa$, we can obtain the following equation:

$$
\partial \theta_{F}(\kappa) / \partial \kappa=2 \kappa(1-3 \kappa)+2\left[S_{F}(\kappa)\left(1-S_{F}^{\backslash}(\kappa)\right)+\kappa S_{F}^{\searrow}(\kappa)\right]+\widetilde{r}_{F}(\kappa) \pi_{F}^{3}(\kappa),
$$

by using (2.2), and (2.4) in (2.5), we can decide that:

$$
\partial \theta_{F}(\kappa) / \partial \kappa=2 \kappa(1-2 \kappa)+2(1-\kappa) S_{F}(\kappa)+\widetilde{r}_{F}(\kappa)\left[S_{F}^{2}(\kappa)+\kappa^{2}(1-\kappa)^{2}-\theta_{F}(\kappa)\right] .
$$

In this way we obtain the complete proof.

As mentioned earlier, the $S R$ classes is important in reliability theory. The next example illustrated the application of theorem 2.1 in reliability theory whereas Theorem 1 in recognizing $\mathbf{S}_{\mathbf{I}}$ class.

Example 2.1: Suppose $Y$ be an Weibull random variable with the density function $g(y)=\beta \theta^{\beta} y^{\beta-1} \exp \left(-(\theta y)^{\beta}\right)$, for $y>0$, and $\beta, \theta>0$. One can easily prove that:

$$
\begin{gathered}
\tilde{r}_{G}(y)=\left(\beta \theta^{\beta} y^{\beta-1} \exp \left(-(\theta y)^{\beta}\right)\right) /\left[1-\exp \left(-(\theta y)^{\beta}\right)\right], \\
S_{G}(y)=\frac{1}{\left[1-\exp \left(-(\theta y)^{\beta}\right)\right]}\left[\left(y^{2} / 2\right)+\left[\frac{\exp \left(-(\theta y)^{\beta}\right)}{\beta \theta^{2}}\left(\sum_{k=1}^{(2 / \beta)-1} \varpi(2)(\theta y)^{2-\beta(k+1)}+(\theta y)^{2-\beta}\right)\right],\right.
\end{gathered}
$$

and

$$
\pi_{G}^{3}(y)=\frac{1}{\left[1-\exp \left(-(\theta y)^{\beta}\right)\right]}\left[y^{4}+4\left(\begin{array}{c}
\frac{\exp \left(-(\theta y)^{\beta}\right)}{\beta \theta^{4}} \\
\times\left(\sum_{k=1}^{(4 / \beta)-1} \varpi(4)(\theta y)^{4-\beta(k+1)}-(\theta y)^{4-\beta}\right)
\end{array}\right)\right]
$$


where $\varpi(j)=(((j / \beta)-1)((j / \beta)-2) \ldots((j / \beta)-k))$. By using (2.4) it can easily check that

$$
S_{G}(y)\left(S_{G}^{\backslash}(y)-1\right)-y S_{G}^{\succ}(y)<y(1-3 y)+\left(\widetilde{r}_{G}(y) \pi_{G}^{3}(y)\right) / 2 .
$$

It follows from Theorem (2.1) that $Y \in \mathbf{S}_{\mathbf{I}}$. Also, according to $Y \in D R H R$ we obtain $Y \in \mathbf{S}_{\mathbf{I}}$.

Introduce the notation

$$
\bar{G}^{(r)}(\kappa)=\int_{\kappa}^{\infty} \bar{G}^{(r-1)} d I(<\infty), \text { for } r=1,2, \ldots
$$

where $\bar{G}^{(0)} \equiv \bar{G}$, and $\Phi_{r} \equiv E\left(Y^{r}\right)$. When normalized, these are the survival functions corresponding to the distribution function $G_{(r)}$ of Smith (1959, pp. 6), furthermore, by using Hall and Wellner (1981, pp. 173) we can prove that $\Phi_{r}=r ! \bar{G}^{(r)}(0)$, for $r=1,2, \ldots$ with $\bar{G}^{(r)}$ finite if and only if $\Phi_{r}$ is finite. Hence $d \bar{G}^{(r)} / d \kappa=-\bar{G}^{(r-1)}$.

The following a list series of inequalities, those provide bounds for $S M P$ and $S R$ functions.

Since

$$
\kappa-S_{G}(\kappa)=E\left[Y^{2} \mid Y \leq \kappa\right]
$$

we have

$$
\left\{\kappa-S_{G}(\kappa)\right\} G(\kappa)=E\left[Y^{2} \cdot I_{(Y<\kappa)}\right]=\Phi_{2}-E\left[Y^{2} \cdot I_{(Y \geq \kappa)}\right] .
$$

It is clear that

$$
E\left[Y^{2} \cdot I_{(Y<\kappa)}\right] \leq \kappa^{2} G(\kappa)
$$

and

$$
E\left[Y^{2} \cdot I_{(Y<\kappa)}\right] \leq \Phi_{2},
$$

and by using Jensen's inequality, we get

$$
E\left[Y^{2} \mid Y \leq \kappa\right] \leq\left(\kappa-m_{G}(\kappa)\right)^{2} .
$$

Further, Hölder's inequality implies that

$$
E\left[Y^{2} \cdot I_{(Y<\kappa)}\right] \leq\left[E\left(Y^{2 w}\right)\right]^{1 / w}[G(\kappa)]^{\left(1-\frac{1}{w}\right)}, \text { for } w>1,
$$

Similarly, $E\left[Y^{2} \cdot I_{(Y \geq \kappa)}\right] \leq \kappa^{2} \bar{G}(\kappa)$ is also true for all $w>1$. In this way we obtain

$$
E\left[Y^{2} \cdot I_{(Y \geq \kappa)}\right] \leq\left[E\left(Y^{2 w}\right)\right]^{1 / w}[\bar{G}(\kappa)]^{\left(1-\frac{1}{w}\right)}, \text { for } w>1 .
$$

It means

$$
\kappa^{2}=\left(E\left(Y^{2 w}\right)\right)^{1 / w}(\bar{G}(\kappa))^{-\frac{1}{w}} .
$$

Furthermore, we can prove that

$$
\theta_{G}(\kappa)=E\left[Y^{4} \mid Y \leq \kappa\right]-\left(E\left[Y^{2} \mid Y \leq \kappa\right]\right)^{2} .
$$

We use below inequalities to obtain bound for $S_{G}(\kappa)$ and $\theta_{G}(\kappa)$.

Proposition 2.1. If $G$ is non-degenerate with $\Phi_{r} \equiv E\left(Y^{r}\right)<\infty$, then, 
(a) $S_{G}(\kappa) \geq \kappa-\left(\Phi_{2} / G(\kappa)\right)$;

(b) $\theta_{G}(\kappa) \leq \Phi_{4}$;

(c) $S_{G}(\kappa) \geq \kappa-\left[\frac{\Phi^{2 w}}{G(\kappa)}\right]^{1 / w}$, for all $\kappa$ and any $w>1$;

(d) $S_{G}(\kappa) \geq \kappa-\left\{\left(\Phi_{2}-\Phi_{2 w}^{1 / w}[\bar{G}(\kappa)]^{\left(1-\frac{1}{w}\right)}\right) / G(\kappa)\right\}$, for $Y \leq \kappa$ and any $w>1$.

Proof. Since $E\left[Y^{2} \cdot I_{(Y<\kappa)}\right]=\Phi_{2}-E\left[Y^{2} \cdot I_{(Y \geq \kappa)}\right]$, it implies that $G(\kappa)\left(\kappa-S_{G}(\kappa)\right) \leq$ $\Phi_{2}$. Then, we finished the proof (a). If

$$
\begin{aligned}
\theta_{G}(\kappa) & =\int_{0}^{\kappa} \frac{\left(\kappa-u^{2}\right)^{2} g(u) d u}{G(\kappa)}-\left[\kappa-E\left[Y^{2} \mid Y \leq \kappa\right]\right]^{2}, \\
& =E\left[Y^{4} \mid Y \leq \kappa\right]-\left(E\left[Y^{2} \mid Y \leq \kappa\right]\right)^{2},
\end{aligned}
$$

it follows that $\theta(\kappa) \leq E\left[Y^{4} \mid Y \leq \kappa\right]$, which leads to complete proof of (b).

Furthermore, we have $G(\kappa)\left\{\kappa-S_{G}(\kappa)\right\}=E\left[Y^{2} \cdot I_{(Y<\kappa)}\right]$, but

$$
E\left[Y^{2} \cdot I_{(Y<\kappa)}\right] \leq\left[E\left(Y^{2 w}\right)\right]^{1 / w}[G(\kappa)]^{\left(1-\frac{1}{w}\right)} .
$$

Therefore,

$$
G(\kappa)\left(\kappa-S_{G}(\kappa)\right) \leq\left[\Phi^{2 w}\right]^{1 / w} G(\kappa)[G(\kappa)]^{-\frac{1}{w}},
$$

which leads to complete proof of (c). Now, we can provide that

$$
E\left[Y^{2} \cdot I_{(Y \geq \kappa)}\right] \leq E\left(Y^{2 w}\right)^{1 / w}[\bar{G}(\kappa)]^{\left(1-\frac{1}{w}\right)}, \text { for } w>1,
$$

In addition, (2.7) implies that

$$
\left\{\kappa-S_{G}(\kappa)\right\} G(\kappa)=E\left[Y^{2} \cdot I_{(Y<\kappa)}\right]=\Phi_{2}-E\left[Y^{2} \cdot I_{(Y \geq \kappa)}\right] .
$$

In this way we obtain

$$
\begin{aligned}
G(\kappa)\left(\kappa-S_{G}(\kappa)\right) & \leq \Phi_{2}-E\left(Y^{2 w}\right)^{1 / w}[\bar{G}(\kappa)]^{\left(1-\frac{1}{w}\right)} \\
& =\Phi_{2}-\Phi_{2 w}^{1 / w}[\bar{G}(\kappa)]^{\left(1-\frac{1}{w}\right)}
\end{aligned}
$$

which leads to complete proof of (d).I 


\section{Preservation Properties of SR Class}

We started by investigating the preservation properties of $S R$ class closed under some reliability operations such as mixture.

For a family of distributions $\{M(\theta \mid \mathbf{X}), x \geq 0\}$ of random samples $\mathbf{X}$ and mixing distribution (prior distribution) $D(\theta)$, it is necessary to evaluate the mixture distribution $(\Psi()$.$) . It obtained as follows$

$$
\Psi(\kappa)=\int_{0}^{\infty} M(\theta \mid \kappa) d D(\theta), \kappa>0 .
$$

In order to verify the validity of $S R$ class will be closed under mixture, we carried out the following theory.

Theorem 3.1. Suppose that $M(\theta \mid \mathbf{X}) \in \mathbf{S}_{\mathbf{I}}$ for any $\theta \in \mathbb{R}^{+}$. Then $\Psi(\kappa) \in \mathbf{S}_{\mathbf{I}}$. Proof. Without loss of generality, suppose that $M(\theta \mid \mathbf{X})=\operatorname{Exp}(\theta)$ and $D(\theta)=\operatorname{Exp}(1)$, that is, $M(\theta \mid \kappa)=M_{\theta}(k)=1-e^{-\theta \kappa}, \kappa>0$, and $D(\theta)=$ $1-e^{-\theta}, \theta>0$, after some algebraic calculations, we have

$$
S_{M_{\theta}}(\kappa)=\frac{\left(\kappa^{2} / 2\right)-\left[(\kappa / \theta) \exp (-\theta \kappa)+\left(1 / \theta^{2}\right)(\exp (-\theta \kappa)-1)\right]}{1-\exp (-\theta \kappa)}, \kappa \in \mathbb{R}^{+}
$$

and

$$
\frac{\int_{0}^{\kappa} x^{3} M_{\theta}(x) d x}{M_{\theta}(\kappa)}=\frac{\left(\kappa^{4} \theta^{4} / 4\right)+\exp (-\theta \kappa)\left(\kappa^{2} \theta^{2}(\theta+3)+6 \theta \kappa+6\right)-6}{(1-\exp (-\theta \kappa)) \theta^{4}},
$$

where $S_{M_{\theta}}(\kappa)$ is SMP of $M_{\theta}$. Suppose

$$
\varsigma(x)=\exp (-\theta x)\left(\left(\frac{4 x^{2} \theta^{2}(\theta+3)+24 \theta x+24}{\theta^{4}}\right)+4\left(x^{2} / \theta\right)+4 x \theta^{-2}\right),
$$

then we have

$$
\frac{4 \int_{0}^{\kappa} x\left(\kappa-x^{2}\right) M_{\theta}(x) d x}{M_{\theta}(\kappa)}=\frac{\kappa^{3} \theta^{4}(2-\kappa)-4 \kappa \theta^{2}-\theta^{4} \varsigma(\kappa)+24}{\theta^{4}[1-\exp (-\theta \kappa)]} .
$$

Hence, $\theta_{M_{\theta}}(\kappa)$ is increasing in $\kappa$, which leads to $M_{\theta} \in \mathbf{S}_{\mathbf{I}}$ for all $\theta \in \mathbb{R}^{+}$.

Now, we checked for the presence of $\Psi \in \mathbf{S}_{\mathbf{I}}$. Firstly, $\Psi(\kappa)$ can be computed by the following equation:

$$
\Psi(\kappa)=\int_{0}^{\infty} M_{\theta}(\kappa) d G(\theta)=1-(1+\kappa)^{-1}, \quad \text { for } \kappa \in \mathbb{R}^{+}
$$

Therefore, the density function of $\Psi$ ( denoted as $\psi$ ) is:

$$
\psi(\kappa)=(1+\kappa)^{-2}, \kappa \in \mathbb{R}^{+},
$$


and the reversed hazard rate (denoted as $\left.\widetilde{r}_{\Psi}(\kappa)\right)$ is

$$
\widetilde{r}_{\Psi}(\kappa)=(1+\kappa)^{-2} /\left[1-(1+\kappa)^{-1}\right], \quad \kappa \in[0, \infty) .
$$

It is evident that $\widetilde{r}_{\Psi}(\kappa)$ is strictly decreasing in $\kappa>0$, this mean that $\widetilde{r}_{\Psi}(\kappa) \in$ $D R H R$. It follows from (2.6) that $\Psi \in \mathbf{S}_{\mathbf{I}}$.

In order to verify the validity of the $\mathbf{S}_{\mathbf{I}}$ is closed under mixing of non-crossing distributions, we provide the following result.

Theorem 3.2. Suppose $\{M(\theta \mid \mathbf{X}), x \geq 0\}$ be a family of life distribution achieving the following requirements:

(i) $M_{\theta}(.) \in \mathbf{S}_{\mathbf{I}}$ for each $\theta \in \mathbb{R}^{+}$.

(ii) $\theta_{1} \neq \theta_{2}$.

(iii) The family $\left\{M_{\theta}(k)\right\}$ is non-crossing function for $\theta_{1}$ and $\theta_{2}$.

Then $\psi(\kappa)$ of $\{M(\theta \mid \mathbf{X}), x \geq 0\}$ is belong to the class $\mathbf{S}_{\mathbf{I}}$.

Proof. Equation (2.5) demonstrate that

$$
S_{\Psi}(\kappa)\left(1-S_{\Psi}^{\searrow}(\kappa)\right)+\kappa S_{\Psi}^{\searrow}(\kappa) \geq-\kappa(1-3 \kappa)-\frac{1}{2} \widetilde{r}_{\Psi}(\kappa) \pi_{\Psi}^{3}(\kappa),
$$

This true when $M_{\theta}(k) \in \mathbf{S}_{\mathbf{I}}$, for all $\kappa \in \mathbb{R}^{+}$. This inequality can be written as

$$
\left(\int_{0}^{\kappa} u \Psi(u) d u\right)^{3} \leq \Psi^{3}(\kappa)\left(\frac{(1-3 \kappa)}{\widetilde{r_{\Psi}}(\kappa)}+\frac{1}{2 \kappa} \pi_{\Psi}^{3}(\kappa)+\frac{1}{\widetilde{r}_{\Psi}(\kappa)}\right)+\frac{1}{\kappa},
$$

where $S_{\Psi}(\kappa)=\int_{0}^{\kappa} x \Psi(x) d x / \Psi(\kappa), \widetilde{r}_{\Psi}(\kappa)=\partial \Psi(\kappa) / \partial \kappa \Psi(\kappa)$ and $\pi_{\Psi}^{3}(\kappa)=$ $4 \int_{0}^{x} y^{3} \Psi(y) d y / \Psi(\kappa)$.

According to (3.1), the property of the non-crossing function and Fubini's theorem we have

$$
\left(\int_{0}^{\kappa} u \Psi(u) d u\right)^{3}=\left(\int_{0}^{\infty}\left[\int_{0}^{\kappa} u F_{\alpha}(u) d G(\alpha)\right] d u\right)^{3} \geq \int_{0}^{\infty}\left[\int_{0}^{\kappa} u F_{\alpha}(u)\right]^{3} d G(\alpha) d u .
$$

From now on we assume that $M(\theta \mid \mathbf{X})=\operatorname{Exp}(\theta)$ and $D(\theta)=\operatorname{Exp}(1)$, and $\kappa=1$. We started by investigating that $\Psi \in \mathbf{S}_{\mathbf{I}}$. Firstly, we can derive that

$$
\begin{aligned}
\left(\int_{0}^{1} u \Psi(u) d u\right)^{3} & =\left(\int_{0}^{1} u\left(1-(1+u)^{-1}\right) d u\right)^{3} \\
& =\left(\ln 2-\frac{1}{2}\right)^{3} \leq \Psi^{3}(1)\left(\frac{-2}{\widetilde{r}_{\Psi}(1)}+\frac{1}{2} \pi_{\Psi}^{3}(1)+\frac{1}{\widetilde{r}_{\Psi}(1)}\right)+1
\end{aligned}
$$

Hence, the mixture $\Psi$ satisfies (3.1), and then $\Psi \in \mathbf{S}_{\mathbf{I}}$. 
Theorem 3.3. The class of lifetime distribution $\mathbf{S}_{\mathbf{I}}$ is not closed under convolution.

Proof. We start with two functions $U(\kappa)$ and $V(\kappa)$, where

$$
U(\kappa)=\left\{\begin{array}{cc}
\kappa, & \text { if } \kappa \in(0,1), \\
0, & \text { if } \kappa \notin(0,1),
\end{array} \quad \text { and } V(x)=\left\{\begin{array}{cr}
1-e^{-\kappa}, & \text { if } \kappa>0 \\
0, & \text { otherwise }
\end{array}\right.\right.
$$

Clearly, $U \in$ unif $(0,1)$, while $V \in \operatorname{Exp}(1)$. Now, consider the following mixture of two distributions $U$ and $V$ :

$$
E_{\theta}(x)=\theta U(x)+\bar{\theta} V(x) \text {, where } \theta \in(0,1) \text { and } \bar{\theta}=1-\theta .
$$

A random variable $T$ is the lifetime of some devices with the distribution function $E_{\theta}$. We started by investigating $E_{\theta} \in \mathbf{S}_{\mathbf{I}}$ or $E_{\theta} \in \mathbf{S}_{\mathbf{D}}$. Hence, by using (2.5) we have

$\partial \theta_{E_{\theta}}(\kappa) / \partial \kappa=2 \kappa(1-3 \kappa)+2\left[S_{E_{\theta}}(\kappa)\left(1-S_{E_{\theta}}^{\backslash}(\kappa)\right)+\kappa S_{E_{\theta}}^{\backslash}(\kappa)\right]+\widetilde{r}_{E_{\theta}}(\kappa) \pi_{E_{\theta}}^{3}(\kappa)$,

where $S_{E_{\theta}}(\kappa)=\int_{0}^{\kappa} x E_{\theta}(x) d x / E_{\theta}(\kappa), \widetilde{r}_{E_{\theta}}(\kappa)=\partial E_{\theta}(\kappa) / \partial \kappa E_{\theta}(\kappa)$ and $\pi_{E_{\theta}}^{3}(\kappa)=$ $4 \int_{0}^{x} y^{3} E_{\theta}(y) d y / E_{\theta}(\kappa)$. Then, we derive

$$
S_{E_{\theta}}(\kappa)=\frac{\theta \frac{\kappa^{3}}{3}+\bar{\theta}\left(\frac{\kappa^{2}}{2}+\kappa e^{-\kappa}+e^{-\kappa}-1\right)}{\theta \kappa+\bar{\theta}\left(1-e^{-\kappa}\right)} \text { and } \widetilde{r}_{E_{\theta}}(\kappa)=\frac{\theta+\bar{\theta}\left(e^{-\kappa}\right)}{\theta \kappa+\bar{\theta}\left(1-e^{-\kappa}\right)}
$$

Furthermore

$$
\pi_{E_{\theta}}^{3}(\kappa)=\frac{\theta \frac{4 \kappa^{5}}{5}+\bar{\theta}\left(\kappa^{4}+4 \kappa^{2} e^{-\kappa}+12 \kappa^{2} e^{-\kappa}+24\left(\kappa e^{-\kappa}+e^{-\kappa}-1\right)\right)}{\theta \kappa+\bar{\theta}\left(1-e^{-\kappa}\right)} .
$$

Additionally, Eq. (2.4) and Eq. (3.3) satisfy the following relation

$$
S_{E_{\theta}}(\kappa)=1-\frac{\left(\theta+\bar{\theta}\left(e^{-\kappa}\right)\right)\left(\theta \frac{\kappa^{3}}{3}+\bar{\theta}\left(\frac{\kappa^{2}}{2}+\kappa e^{-\kappa}+e^{-\kappa}-1\right)\right)}{\left(\theta \kappa+\bar{\theta}\left(1-e^{-\kappa}\right)\right)\left(\theta \kappa+\bar{\theta}\left(1-e^{-\kappa}\right)\right)} .
$$

Thus by setting $(\kappa=1$, and $\theta=1 / 2)$, we have $\theta_{E_{\theta}}(\kappa)$ is decreasing. Hence, we can decide that $E_{\theta}(\kappa) \notin \mathbf{S}_{\mathbf{I}}$ i.e. $E_{\theta}(\kappa) \in \mathbf{S}_{\mathbf{D}}$.

Let $F_{1}$ and $F_{2}$ be two independent distributions function of $T_{1}$ and $T_{2}$. Suppose $F_{1}$ and $F_{2}$ are $\mathbf{S}_{\mathbf{I}}$ and the distribution of $T_{1}+T_{2}$ can be represented as

$$
F_{T_{1}+T_{2}}(\kappa)=\left(F_{1} * F_{2}\right)(\kappa)=\int_{0}^{\kappa} F_{1}(\kappa-u) d F_{2}(u), \quad \kappa \in \mathbb{R}^{+} .
$$

Let us take $T_{1}, T_{2} \in E_{\theta}(x)$ and the $G$ is convolution of $E_{\theta}$, i.e. $C(\kappa)=$ $E_{\theta} * E_{\theta}(\kappa)$. Equations (3.2) and (3.4) demonstrate that:

$$
e_{\theta}(\kappa)=\theta+\bar{\theta} \exp (-\kappa)
$$


where $e_{\theta}($.$) is the density function of E_{\theta}($.$) , and$

$$
C(\kappa)=0.5 \theta^{2} \kappa^{2}+\bar{\theta}[\alpha-\beta]
$$

where

$$
\alpha=2 \theta(\exp (-\kappa)+\kappa-1), \text { and } \beta=\bar{\theta} \exp (-\kappa)(1-\kappa)+\bar{\theta}
$$

Thus, $S M P$ of $C$ can present as follow:

$S_{C}(\kappa)=\frac{\frac{2}{3} \kappa^{3} \theta^{2}-\frac{3}{2} \kappa^{2} \theta^{2}+0.125 \kappa^{4} \theta^{2}+(2 \theta \bar{\theta}-\overline{\theta \theta}) \varepsilon_{\kappa}+\overline{\theta \theta}\left(\kappa^{2} \exp (-\kappa)+2 \varepsilon_{\kappa}\right)}{0.5 \theta^{2} \kappa^{2}+\bar{\theta}[\alpha-\beta]}$.

Moreover,

$$
\widetilde{r}_{C}(\kappa)=\frac{\theta+\bar{\theta} \exp (-\kappa)}{0.5 \theta^{2} \kappa^{2}+\bar{\theta}[\alpha-\beta]},
$$

and

$$
\pi_{C}^{3}(\kappa)=\frac{4\left(\rho(\kappa)+\eta(\kappa)+0.5 \theta^{2} \frac{\kappa^{6}}{6}+2 \theta \bar{\theta} \frac{\kappa^{5}}{5}-2 \theta \bar{\theta} \frac{\kappa^{4}}{4}-\overline{\theta \theta} \frac{\kappa^{4}}{4}\right)}{0.5 \theta^{2} \kappa^{2}+\bar{\theta}[\alpha-\beta]} .
$$

where $S_{C}(\kappa)=\int_{0}^{\kappa} x C(x) d x / C(\kappa), \widetilde{r}_{C}(\kappa)=\partial C(\kappa) / \partial \kappa C(\kappa)$, and $\pi_{C}^{3}(\kappa)=$ $4 \int_{0}^{x} y^{3} C(y) d y / C(\kappa), \varepsilon_{\kappa}=(-\kappa \exp (-\kappa)-\exp (-\kappa)+1), \rho(\kappa)=(1-\theta)^{2}($ $\left.24-24 \kappa e^{-\kappa}-16 \kappa^{2} e^{-\kappa}-\kappa^{4} e^{-\kappa}-24 e^{-\kappa}\right)$ and $\eta(\kappa)=\left(2 \theta(1-\theta)-(1-\theta)^{2}\right)(6-$ $\left.6 \kappa e^{-\kappa}-4 \kappa^{2} e^{-\kappa}-6 e^{-\kappa}\right)$. Then we can decide that $\partial \theta_{C}(\kappa) / \partial \kappa$ is $\mathbf{I}$. Thus $C \in \mathbf{S}_{\mathbf{I}}$. Therefore, $T_{1}$ and $T_{2} \notin \mathbf{S}_{\mathbf{I}}$. But their convolution $C$ is belong to $\mathbf{S}_{\mathbf{I}}$. It mean $\mathbf{S}_{\mathbf{I}}$ is not closed under convolution, in general. Hence, the proof is complete.

\section{Properties of SR Ordering Under Operations}

In this section, we focus our attention on a new stochastic comparison defined in terms of $S R$ function. The next theorem provides that $\leq_{\theta(t)}$ order lies between $\leq_{R H R}$ and $\leq_{\theta(t)}$.

Theorem 4.1. Let $X_{1} \geq 0$ and $X_{2} \geq 0$ be two continuous random variables, with the distribution functions $F_{1}($.$) and F_{2}($.$) , and let \alpha^{i}(t)=\int_{0}^{t} v^{i} F_{1}(v) d v$ $/ F_{1}(t)$ and $\beta^{i}(t)=\int_{0}^{t} v^{i} F_{2}(v) d v / F_{2}(t)$. Then we have

1. $X_{1} \leq_{R H R} X_{2} \Rightarrow X_{1} \leq_{\theta(t)} X_{2}$,

2. $X_{1} \leq_{S M P} X_{2} \Rightarrow X_{1} \leq_{\theta(t)} X_{2}$,

3. $X_{1} \leq_{\theta(t)} X_{2} \Rightarrow X_{1} \leq_{V P} X_{2}$. 
Proof: (1) By definition of $\leq_{R H R}$ we can obtain

$$
\int_{0}^{t} \frac{\alpha^{0}(x) F_{1}(x)}{F_{1}(t)}-\frac{\beta^{0}(x) F_{2}(x)}{F_{2}(t)} d x \geq 0 \text {, for all } t \in \mathbb{R}^{+} .
$$

which means that $X_{1} \leq_{\theta(t)} X_{2}$.

(2). Note that $X_{1} \leq_{S M P} X_{2}$ implies

$$
\frac{\alpha^{3}(t) F_{1}(t)}{\beta^{3}(t) F_{2}(t)} \text { is increasing in } t \text {. }
$$

which, by using the same type of argument as used in proving theorem 3.1 of Nanda et al. (2003), gives

$$
\frac{\int_{0}^{t} \alpha^{0}(u) F_{1}(u) d u}{\int_{0}^{t} \beta^{0}(u) F_{2}(u) d u} \text { is increasing in } t \in \mathbb{R}^{+} .
$$

Hence, by (1.1), we have our result.

As follows from the theorem shown above, the next example indicates that $X \leq_{\theta(t)} Y \nrightarrow X \leq_{R H R} Y$ and $X \leq_{\theta(t)} Y \nrightarrow X \leq_{S M P} Y$. In addition, $X \leq_{V P} Y$ $\nrightarrow X \leq_{\theta(t)} Y$.

Example 4.1 : Let $X_{1}$ and $X_{2}$ be to non-negative random variables with the distribution functions $F_{1}$, and $F_{2}$, respectively, which are given by

$$
F_{1}(u)=\left\{\begin{array}{cc}
\frac{u}{2} & 0 \leq u \leq 2 \\
1 & u>2
\end{array}\right.
$$

and

$$
F_{2}(u)=\left\{\begin{array}{cc}
\frac{u^{2}}{2} & 0 \leq u \leq 1 \\
\frac{u^{2}+2}{6} & 1<u \leq 2 \\
1 & u>2
\end{array}\right.
$$

Then, after some algebraic calculations, we deduce that

$$
\frac{\int_{0}^{t} u\left(t-u^{2}\right) F_{1}(u) d u}{\int_{0}^{t} u\left(t-u^{2}\right) F_{2}(u) d u}= \begin{cases}\frac{8}{10 \frac{5-3 t}{3 t-2 t^{2}}} & 0 \leq t \leq 1 \\ \frac{t^{4}(10-6 t)}{\left(\frac{5 t^{5}}{2}-\frac{5 t^{6}}{3}+10 t^{3}-5 t^{4}-t \frac{25}{2}+\frac{20}{3}\right)} & 1<t \leq 2 \\ 1 & t>2\end{cases}
$$

and

$$
\frac{\int_{0}^{t} \alpha^{0}(u) F_{1}(u) d u}{\int_{0}^{t} \beta^{0}(u) F_{2}(u) d u}=\left\{\begin{array}{cc}
\frac{2}{t} & 0 \leq t \leq 1 \\
\frac{6 t}{t^{2}+12} & 1<t \leq 2 \\
1 & x>t
\end{array}\right.
$$

By (4.1) we can conclude that $X_{1} \leq_{\theta(t)} X_{2}$ does not hold in this case. While in parallel, Kayid and Izadkhah (2014, pp.595) showed in their counterexample 1 that $X_{1} \leq_{S M P} X_{2}$. Also, (4.2) is increasing in $t$, and hence $X_{1} \leq_{V P} X_{2}$. 
The next result provides useful characterization of the $S R$ order.

Theorem 4.2. Suppose $X_{1} \in \mathbb{R}^{+}$and $X_{2} \in \mathbb{R}^{+}$be two continuous random variables with distribution functions $F_{1}$ and $F_{2}$, respectively, and let $\alpha^{i}(x)=$ $\int_{0}^{x} u^{i} F_{1}(u) d u / F_{1}(x)$ and $\beta^{i}(x)=\int_{0}^{x} u^{i} F_{2}(u) d u / F_{2}(x)$, hence $X_{1} \leq_{S M P}$ $X_{2}$, if $X_{1} \leq_{\theta(t)} X_{2}$ holds, therefore

$$
m_{F_{1}}(t)-m_{F_{2}}(t) \geq\left\{S_{F_{1}}(t)-S_{F_{2}}(t)\right\} / t,
$$

and

$$
X_{1} \leq_{M P} X_{2} .
$$

Proof. If $X_{1} \leq_{\theta(t)} X_{2}$ holds, we obtain

$t F_{2}(t) \int_{0}^{t} \alpha^{i}(u) F_{1}(u) d u-t F_{1}(t) \int_{0}^{t} \beta^{i}(u) F_{2}(u) d u \geq F_{1}(t) F_{2}(t)\left(\alpha^{3}(t)-\beta^{3}(t)\right)$.

It evident that

$$
\int_{0}^{t}(t-u)\left\{F_{1}(u) F_{2}(t)-F_{2}(u) F_{1}(t)\right\} d u \geq \int_{0}^{t} u^{3}\left\{F_{1}(u) F_{2}(t)-F_{2}(u) F_{1}(t)\right\} d u / t,
$$

and

$$
\begin{gathered}
t^{2}\left(m_{F_{1}}(t)-m_{F_{2}}(t)\right)+t\left(S_{F_{2}}(t)-S_{F_{1}}(t)\right) \\
\geq \int_{0}^{t} x^{3} F_{1}(x) d x / F_{1}(t)-\int_{0}^{t} x^{3} F_{2}(x) d x / F_{2}(t),
\end{gathered}
$$

since $X_{1} \leq_{S M P} X_{2}$, Thus the following result is obtained:

$$
\int_{0}^{t} u^{3} F_{1}(u) F_{2}(t)-u^{3} F_{2}(u) F_{1}(t) d u \geq 0
$$

consequently,

$$
t m_{F_{1}}(t)-t m_{F_{2}}(t) \geq S_{F_{1}}(t)-S_{F_{2}}(t),
$$

which is equivalent to the required statement.I

In next result develop some preservation of $S M P$ and $S R$ orders and $\mathbf{S}_{\mathbf{I}}$ class.

Proposition 4.1. Let $X_{1} \in \mathbb{R}^{+}$be continuous random variable with distribution function $F_{1}$ and SMP function $S_{F_{1}}(t)$, hence $S_{F_{1}}(t)$ is increasing in $t$ if $X_{1} \leq_{S M P} X_{1}+X_{2}$ for any continuous random variable $X_{2} \in \mathbb{R}^{+}$independent of $X_{1}$. 
Proof: By apply Fubini's theorem and using change of order double integration, it is obvious that

$$
\begin{aligned}
S_{X_{1}+X_{2}}(t) & =\frac{\int_{0}^{t} \int_{0}^{y} y F_{1}(y-v) d F_{2}(v) d y}{\int_{0}^{t} F_{1}(t-v) d F_{2}(v)}, \\
& =\frac{\int_{0}^{t} \int_{0}^{t-v}(y+v) F_{1}(y) d y d F_{2}(v)}{\int_{0}^{t} F_{1}(t-v) d F_{2}(v)} \\
& =\frac{\int_{0}^{t} F_{1}(t-v) S_{X}(t-v) d F_{2}(v)+\int_{0}^{t} v F_{1}(t-v) m_{X_{1}}(t-v) d F_{2}(v)}{\int_{0}^{t} F_{1}(t-v) d F_{2}(v)} \\
& \leq S_{X_{1}}(t)+\frac{\int_{0}^{t} v F_{1}(t-v) m_{X_{1}}(t-v) d F_{2}(v)}{\int_{0}^{t} F_{1}(t-v) d F_{2}(v)}
\end{aligned}
$$

This is true for $S_{F_{1}}(t)$ is increasing in $t \in \mathbb{R}^{+}$. Therefore, we shall write the above expression as

$$
S_{X_{1}+X_{2}}(t) \geq S_{X_{1}}(t),
$$

when $a=\int_{0}^{t} u F_{1}(t-u) m_{X_{1}}(t-u) d F_{2}(u) / \int_{0}^{t} F_{1}(t-u) d F_{2} u$ and $a \geq S_{X_{1}+X_{2}}(t)$. Hence, we have the required results.

Theorem 4.3. Let $X_{1} \in \mathbb{R}^{+}$be continuous random variable with distribution function $F_{1}$ and SR function $\theta_{F_{1}}(t)$, then $X_{1} \in \mathbf{S}_{\mathbf{I}}$ if $X_{1} \leq_{\theta(t)} X_{1}+X_{2}$ for any continuos random variable $X_{2}$, that is independent of $X_{1}$.

Proof: Based on Fubini's theorem and using change of order double integration, it is clear that

$$
\begin{aligned}
\theta_{X_{1}+X_{2}}(t)+S_{X_{1}+X_{2}}^{2}(t)= & (t(1-t))^{2}+\frac{4 t \int_{0}^{t} \int_{0}^{y} \int_{0}^{x} F_{1}(x-v) d F_{2}(v) d x d y}{\int_{0}^{t} F_{1}(t-v) d F_{2}(v)} \\
& -\frac{4 \int_{0}^{t} \int_{0}^{v} z^{3} F_{1}(z-v) d F_{2}(v) d z}{\int_{0}^{t} F_{1}(t-v) d F_{2}(v)} \\
= & (t(1-t))^{2}+\frac{4\left[\begin{array}{c}
t \int_{0}^{t} \int_{0}^{t-v} \int_{0}^{y-v} F_{1}(x) d x d y d F_{2}(v) \\
-\int_{0}^{t} \int_{v}^{t} z^{3} F_{1}(z-v) d z d F_{2}(v)
\end{array}\right]}{\int_{0}^{t} F_{1}(t-v) d F_{2}(v)} .
\end{aligned}
$$

This is true for $\theta_{F_{1}}(t)$ increasing in $t \in \mathbb{R}^{+}$. According to (4.3) we obtain

$$
(t(1-t))^{2}+\frac{\int_{0}^{t} F_{1}(t-v)\left[\begin{array}{c}
\theta_{X_{1}}(t-v)+S_{X_{1}}^{2}(t-v) \\
-((t-v)(1-(t-v)))^{2}
\end{array}\right] d F_{2}(v)}{-4 \int_{0}^{t} \int_{0}^{t-v}\left[(y+v)^{3}-\frac{1}{4} y^{3}\right] F_{1}(y) d y d F_{2}(v)} \leq \theta_{X_{1}}(t)+S_{X_{1}}^{2}(t),
$$

where

$$
\int_{0}^{t} \int_{0}^{t-v}\left[4 v^{3}+12 v y^{2}+12 y v^{2}\right] F_{1}(y) d y d F_{2}(v) \geq \int_{0}^{t} \int_{0}^{t-v} y^{3} F_{1}(y) d y d F_{2}(v) .
$$


By apply proposition (4.1) we can prove that $S_{X_{1}+X_{2}}^{2}(t) \geq S_{X_{1}}^{2}(t)$. This completes the proof.

Theorem 4.4. Suppose $X_{1} \in \mathbb{R}^{+}$and $X_{2} \in \mathbb{R}^{+}$are two random variables with distribution functions $U_{1}$ and $U_{2}$ respectively, and let $\psi$ is strictly increasing and convex upwards and $\psi(0)=0$. If $X_{1} \leq_{\theta(t)} X_{2}$, then we have $\psi\left(X_{1}\right) \leq_{\theta(t)}$ $\psi\left(X_{2}\right)$.

Proof: Let $\psi$ is differentiable with derivative $\psi \backslash$. If $X_{1} \leq_{\theta(t)} X_{2}$ then for any $t>0$, we have

$$
\begin{aligned}
\theta_{U_{1}}(t) & =\frac{4\left[t \int_{0}^{t} \int_{0}^{x} U_{1}(u) d u d x-\int_{0}^{t} v^{3} U_{1}(v) d v\right]}{U_{1}(t)}-\left(\int_{0}^{t} \frac{u U_{1}(v) d v}{U_{1}(t)}\right)^{2} \\
& \geq \frac{4\left[t \int_{0}^{t} \int_{0}^{x} U_{2}(u) d u d x-\int_{0}^{t} v^{3} U_{2}(v) d v\right]}{U_{2}(t)}-\left(\int_{0}^{t} \frac{u U_{2}(u) d u}{U_{2}(t)}\right)^{2}=\theta_{U_{2}}(t) .
\end{aligned}
$$

It lead to

$$
\begin{aligned}
& 4 \psi^{-1}(t)\left[\frac{\int_{0}^{\psi^{-1}(t)} \int_{0}^{\psi^{-1}(v)} U_{1}(u) d u d v}{U_{1}\left(\psi^{-1}(t)\right)}-\frac{\int_{0}^{\psi^{-1}(t)} \int_{0}^{\psi^{-1}(v)} U_{2}(u) d u d v}{U_{2}\left(\psi^{-1}(t)\right)}\right] \\
& -4\left[\frac{\int_{0}^{\psi^{-1}(t)} x^{3} U_{1}(x) d x}{U_{1}\left(\psi^{-1}(t)\right)}-\frac{\int_{0}^{\psi^{-1}(t)} x^{3} U_{2}(x) d x}{U_{2}\left(\psi^{-1}(t)\right)}\right] \\
& \geq \quad\left[\left(\int_{0}^{\psi^{-1}(t)} \frac{u U_{1}(u) d u}{U_{1}\left(\psi^{-1}(t)\right)}\right)^{2}-\left(\int_{0}^{\psi^{-1}(t)} \frac{u U_{2}(u) d u}{U_{2}\left(\psi^{-1}(t)\right)}\right)^{2}\right]
\end{aligned}
$$

According Kayid and Izadkhah (2014, Theorem 5, pp. 596) we can prove

$$
\begin{aligned}
X_{1} & \leq S_{S M P} X_{2} \Rightarrow \psi\left(X_{1}\right) \leq_{S M P} \psi\left(X_{2}\right) \\
& \Rightarrow S_{U_{1}}^{2}(t) \geq S_{U_{2}}^{2}(t) \Rightarrow S_{U_{1}}^{2}(\psi(t)) \geq S_{U_{2}}^{2}(\psi(t))
\end{aligned}
$$

since $t \geq 0$ and $S_{U_{1}}(t)$ and $S_{U_{2}}(t)$ are non-negative functions. According Mahdy 
(2012) we can decide that

$$
\begin{aligned}
& \frac{\int_{0}^{\psi^{-1}(t)} \int_{0}^{\psi^{-1}(v)} U_{1}(u) d u d v}{U_{1}\left(\psi^{-1}(t)\right)}-\frac{\int_{0}^{\psi^{-1}(t)} \int_{0}^{\psi^{-1}(v)} U_{2}(u) d u d v}{U_{2}\left(\psi^{-1}(t)\right)} \\
= & \frac{\int_{0}^{t} \int_{0}^{v} U_{1}\left(\psi^{-1}(u)\right) d u d v}{U_{1}\left(\psi^{-1}(t)\right)}-\frac{\int_{0}^{t} \int_{0}^{v} U_{2}\left(\psi^{-1}(u)\right) d u d v}{U_{2}\left(\psi^{-1}(t)\right)} .
\end{aligned}
$$

In addition, $X_{1} \leq_{\theta(t)} X_{2}$ implies that,

$$
\frac{\int_{0}^{t} x^{3} U_{1}(x) d x}{U_{1}(t)} \leq \frac{\int_{0}^{t} x^{3} U_{2}(x) d x}{U_{2}(t)}
$$

where $t \in \mathbb{R}^{+}$. It is clear that

$$
\frac{\int_{0}^{\psi^{-1}(t)} x^{3} U_{1}(x) d x}{U_{1}\left(\psi^{-1}(t)\right)} \leq \frac{\int_{0}^{\psi^{-1}(t)} x^{3} U_{2}(x) d x}{U_{2}\left(\psi^{-1}(t)\right)},
$$

and

$$
\int_{0}^{\psi^{-1}(t)}\left[\frac{v^{3} U_{1}(v)}{U_{1}\left(\psi^{-1}(t)\right)} \leq \frac{v^{3} U_{2}(v)}{U_{2}\left(\psi^{-1}(t)\right)}\right] d v \leq 0 .
$$

On the other hand, $\psi\left(X_{1}\right) \leq_{\theta(t)} \psi\left(X_{2}\right)$ iff, for all $t>0$ and $\psi(0)=0$,

$$
\frac{\int_{0}^{t} v^{3} \operatorname{Pr}\left(\psi\left(X_{1}\right) \leq v\right) d v}{\operatorname{Pr}\left(\psi\left(X_{1}\right) \leq v\right)} \leq \frac{\int_{0}^{t} v^{3} \operatorname{Pr}\left(\psi\left(X_{2}\right) \leq v\right) d v}{\operatorname{Pr}\left(\psi\left(X_{2}\right) \leq v\right)},
$$

since

$$
\operatorname{Pr}\left(\psi\left(X_{1}\right) \leq x\right)=\operatorname{Pr}\left(X_{1} \leq \psi^{-1}(x)\right)=U_{1}\left(\psi^{-1}(x)\right),
$$

therefore,

$$
\frac{\int_{0}^{t} x^{3} U_{1}\left(\psi^{-1}(x)\right) d x}{U_{1}\left(\psi^{-1}(t)\right)} \leq \frac{\int_{0}^{t} x^{3} U_{2}\left(\psi^{-1}(x)\right) d x}{U_{2}\left(\psi^{-1}(t)\right)} .
$$

If we let $\theta=\psi^{-1}(x) \Rightarrow x=\psi(\theta) \Rightarrow d x / d \theta=\psi^{/}(\theta)$ then we obtain

$$
\frac{\int_{0}^{\psi^{-1}(t)} \psi^{3}(\theta) \psi^{\prime}(\theta) U_{1}(\theta) d \theta}{U_{1}\left(\psi^{-1}(t)\right)} \leq \frac{\int_{0}^{\psi^{-1}(t)} \psi^{3}(\theta) \psi^{\prime}(\theta) U_{2}(\theta) d \theta}{U_{2}\left(\psi^{-1}(t)\right)},
$$


and

$$
\int_{0}^{\psi^{-1}(t)} \beta(x)\left[\frac{x^{3} U_{1}(\theta) d \theta}{U_{1}\left(\psi^{-1}(t)\right)}-\frac{x^{3} U_{2}(\theta) d \theta}{U_{2}\left(\psi^{-1}(t)\right)}\right] \leq 0,
$$

where $\beta(s)=\psi^{3}(s) \psi^{\prime}(s) / s^{3}$. Its obvious that, if $\psi(s)$ is non-negative and decreasing, we can conclude that $\psi^{3}(s) / s^{3}$ is decreasing in $s$, it is implies that $\beta(s)$ is decreasing in $s$. Therefore, the complete proof is given by apply lemma 7.1(b) of Barlow and Proschan (1981).

\section{$5 \quad$ Reliability Applications of SR Ordering}

In the following results, we explore the possibility of apply a new techniques in statistical reliability theory. Let $U_{1}, U_{2}, \ldots, U_{n}$ denote the component lifetimes of the system and assume that $U_{1: n}, U_{2: n}, \ldots, U_{n: n}$ represent the ordered lifetimes of the components.

\subsection{Order Statistics}

Theorem 5.1. Let $U_{1}, U_{2}, \ldots, U_{n}$ and $V_{1}, V_{2}, \ldots, V_{n}$ be independent and identically distributed (i.i.d) copies of $U$ and $V$, with distribution functions $\mathbb{H}_{1}$ and $\mathbb{H}_{2}$ respectively. If $U_{n: n} \leq_{\theta(t)} V_{n: n}$, then we have $U_{i} \leq_{\theta(t)} V_{i}$.

Proof. Let $U_{n: n} \leq_{\theta(t)} V_{n: n}$ hold. Then we implies that

$t \int_{0}^{t} \int_{0}^{x}\left\{\mathbb{H}_{1}^{n}(u) \mathbb{H}_{2}^{n}(t)-\mathbb{H}_{2}^{n}(u) \mathbb{H}_{1}^{n}(t)\right\} d u d x \geq \int_{0}^{t} x^{3}\left\{\mathbb{H}_{1}^{n}(x) \mathbb{H}_{2}^{n}(t)-\mathbb{H}_{2}^{n}(x) \mathbb{H}_{1}^{n}(t)\right\}$

Since,

$$
k(u)=\left[\sum_{i=1}^{n}\left[\mathbb{H}_{2}^{n-i}(t) \mathbb{H}_{1}^{n-i}(u)\right]\left[\mathbb{H}_{1}^{i-1}(t) \mathbb{H}_{2}^{i-1}(u)\right]\right]^{-1},
$$

is non-negative and decreasing in $u \geq 0$ for any $t>0$. Now, we can derive

$$
\mathbb{H}_{2}(t) \mathbb{H}_{1}(u)-\mathbb{H}_{1}(t) \mathbb{H}_{2}(u)=\left\{\mathbb{H}_{1}^{n}(u) \mathbb{H}_{2}^{n}(t)-\mathbb{H}_{2}^{n}(u) \mathbb{H}_{1}^{n}(t)\right\} k(u),
$$

and

$t \int_{0}^{t} \int_{0}^{x}\left\{\mathbb{H}_{1}(u) \mathbb{H}_{2}(t)-\mathbb{H}_{2}(u) \mathbb{H}_{1}(t)\right\} d u d x \geq \int_{0}^{t} v^{3}\left\{\mathbb{H}_{1}(v) \mathbb{H}_{2}(t)-\mathbb{H}_{2}(v) \mathbb{H}_{1}(t)\right\} d v$ for any $t>0$

based on Lemma 7.1(b) of Barlow and Proschan (1981). It lead to $U_{i} \leq_{\theta(t)} V_{i}$,

for $i \in \mathbb{N}$.I 


\subsection{Discrete SR order}

Definition 5.1. Suppose $X \in \mathbb{R}^{+}$and $Y \in \mathbb{R}^{+}$be two random variables, with distribution functions $F_{X}$ and $F_{Y}$, and discrete strong mean past lifetime $S_{d, X}(x)$ and $S_{d, Y}(x)$ respectively. Then, it is said that $X$ is smaller than or equal $Y$ in discrete $\mathrm{SR}$ order $\left(X \leq_{d-\theta(x)} Y\right)$, if

$$
S_{d, X}(x)+\frac{\sum_{j=l_{x}}^{x-1} j^{3} F_{X}(j)}{2 x F_{X}(x)} \geq S_{d, Y}(x)+\frac{\sum_{j=l_{x}}^{x-1} j^{3} F_{Y}(j)}{2 x F_{Y}(x)}, \text { for } x \in N_{+} .
$$

Given a sequence of absolutely continuous non-negative random variables $Z_{1}, Z_{2}, \ldots$ are i.i.d. random variables with common cumulative distribution function $F_{Z_{1}}$ and a common density function $f_{Z_{1}}$. Let $\mathbb{M}_{1} \in N^{+}$and $\mathbb{M}_{2} \in N^{+}$ be two random variables which are independent of the $Z_{i}$ 's. Denote $Z_{1: \mathbb{M}_{j}} \equiv$ $\min \left\{Z_{1}, Z_{2}, \ldots, Z_{\mathbb{M}_{j}}\right\}$ and $Z_{\mathbb{M}_{j}: \mathbb{M}_{j}} \equiv \max \left\{Z_{1}, Z_{2}, \ldots, Z_{\mathbb{M}_{j}}\right\}, j=1,2$. Below we consider the $S R$ order between two such extreme order statistics.

Theorem 5.2. Let $\mathbb{M}_{1} \leq_{d-\theta(z)} \mathbb{M}_{2}$, then we have $Z_{\mathbb{M}_{1}: \mathbb{M}_{1}} \nless_{\theta(z)} Z_{\mathbb{M}_{2}: \mathbb{M}_{2}}$.

Proof. The density function of $Z_{\mathbb{M}_{j}: \mathbb{M}_{j}}$ is given by

$$
f_{Z_{\mathbb{M}_{j}: \mathbb{M}_{j}}}(z)=\sum_{n=1}^{\infty} n F_{Z_{1}}^{n-1}(z) f_{Z_{1}}(z) \operatorname{Pr}\left(\mathbb{M}_{j}=n\right),
$$

and the distribution function of $Z_{\mathbb{M}_{j}: \mathbb{M}_{j}}$ which is given by

$$
H_{\mathbb{M}_{j}: \mathbb{M}_{j}}(z)=\sum_{n=1}^{\infty} F^{n}(z) \operatorname{Pr}\left(\mathbb{M}_{j}=n\right), \text { for all } z>0
$$

for all $z>0$, and $n \in N$. Thus, by Shaked \& Shanthikumar (2007), we obtain

$$
\begin{aligned}
\psi(z, j) & =\int_{0}^{z} \int_{0}^{y}\left\{H_{\mathbb{M}_{j}: \mathbb{M}_{j}}(u)-y^{2} H_{\mathbb{M}_{j}: \mathbb{M}_{j}}(y)\right\} d u d y \\
& =\sum_{n=1}^{\infty} \varphi(z, n) \tau(n, j), \text { for all } z>0 \text { and } j=1,2,
\end{aligned}
$$

where $\varphi(z, n)=\int_{0}^{z} \int_{0}^{y}\left\{F^{n}(u)-y^{2} F^{n}(y)\right\} d u d y$ and in addition, $\tau(n, j)=$ $\operatorname{Pr}\left(\mathbb{M}_{j}=n\right)$. Let $k(n, j)=\sum_{i=n}^{\infty} \tau(i, j)$, for each $n \in N$ and $j=1,2$. We observe that $\mathbb{M}_{1} \leq_{L R} \mathbb{M}_{2}$, implies $\mathbb{M}_{1} \leq_{\theta(z)} \mathbb{M}_{2}$ by using Theorem 1.C.1 on page 43 of Shaked and Shanthikumar (2007), and Li and Zhang (2008). If $\mathbb{M}_{1} \leq{ }_{L R} \mathbb{M}_{2}$, and thus $k(n, j)$ is $T P_{2}$ in $(n, j) \in N \times\{1,2\}$. But it is not hard to see that $\varphi(z, n)$ isn't $T P_{2}$ in $(n, j) \in N \times\{1,2\}$, where $\operatorname{det}[\varphi(z, n)]$ is negative for any strictly increasing convex function and $z F^{n}(z)-\int_{0}^{z} u f_{Z_{\mathbb{M}_{j}: \mathbb{M}_{j}}}(u) d u \leq z^{3} F^{n}(z)$, for $z \in R^{+}$. Also, therefore, by the basic composition formula (Theorem 5.1 on page 123 of Karlin (1968)) we can be seen that $\psi(z, j)$ is not $T P_{2}$ in $z \geq 0$ and $j \in\{1,2\}$. That is, $Z_{\mathbb{M}_{1}: \mathbb{M}_{1}} \Varangle_{\theta(z)} Z_{\mathbb{M}_{2}: \mathbb{M}_{2}} . \mathbf{I}$ 


\subsection{Inactivity time of random at a random}

Let $U$ and $V$ be two the lifetimes of system $C_{1}$ and system $C_{2}$, respectively. Then the residual lifetime at random time $V$ can be represented as

$$
U_{V}=U-V \mid U>V .
$$

It represents the residual life of system $C_{1}$ at a time when system $C_{2}$ fails. Dequan and Jinhua (2000) established a number of stochastic orders for $U_{V}$ under a lot of assumptions of $U$ and $V$. In addition, Misra et al. (2008) studied adequate permissions for log-concavity and log-convexity of the residual life at random time. Stochastic orders are conducted under particular permissions on the concerned total life and random time and their preservation properties are established by Li and Zuo (2004). Dewan and Khaledi (2014) presented now stochastic orders between multivariate residual lifetime at random time and studies some characterizations. Furthermore suppose $G($.$) be distribution$ function of random variable $V$ and $V_{i} / s$ hold for $V$ as well. Moreover, let $U$ and $V$ are independent. The random variable $U_{(V)}=V-U \mid U \leq V$ is called the inactivity time of $U$ at a random time $V$. It has the distribution function

$$
G_{U_{(V)}}(x)=\int_{0}^{\infty}\left[G_{1}(y)-G_{1}(y-x)\right] d G_{2}(y) / \int_{0}^{\infty} G_{1}(y) d G_{2}(y), x \geq 0 .
$$

when $U$ and $V$ are $U$-independent. The following results shows that $U_{(t)} \leq_{S M P}$ $U$ does imply $U_{(V)} \leq_{S M P} U$, in addition, $U_{(t)} \leq_{\theta(t)} U$ does imply $U_{(V)} \leq_{\theta(t)} U$.

Theorem 5.3: $U_{(V)} \leq_{S M P} U$ for any $V$ that is d-independent of $U$ iff $U_{(t)} \leq_{S M P} U$ for all $t \geq 0$.

Proof. When $U_{(t)} \leq_{S M P} U$ for all $t, u \geq 0$, we obtain the following:

$$
\frac{\int_{0}^{u} v\left[G_{1}(t)-G_{1}(t-v)\right] d v d G_{2}(y)}{G_{1}(t)-G_{1}(t-u)} \geq \frac{\int_{0}^{u} v G_{1}(v) d v}{G_{1}(u)} .
$$

From this we deduce that

$$
\begin{aligned}
\frac{\int_{0}^{\infty} \int_{0}^{u} v\left[G_{1}(t)-G_{1}(t-v)\right] d v d G_{2}(y)}{\int_{0}^{\infty}\left[G_{1}(t)-G_{1}(t-u)\right] d G_{2}(t)} & \geq \frac{\int_{0}^{\infty} \frac{G_{1}(t)-G_{1}(t-u)}{G_{1}(u)} \int_{0}^{u} x G_{1}(x) d x d G_{2}(y)}{\int_{0}^{\infty} G_{1}(t) d G_{2}(t)}, \\
& =\frac{\int_{0}^{\infty}\left[G_{1}(t)-G_{1}(t-u)\right] S(u) d G_{2}(y)}{\int_{0}^{\infty}\left[G_{1}(t)-G_{1}(t-u)\right] d G_{2}(t)} \\
& =S(u), \text { for any } u \geq 0
\end{aligned}
$$

by using (5.1), we can decide that $U_{(V)} \leq_{S M P} U$. Further, let $U_{(V)} \leq_{S M P} U$ holds for all value of $y$ and let $y$ equal to constant then $U_{(t)} \leq_{S M P} U$ for all $t \geq 0$..

Proposition 5.1. $U_{(V)} \leq_{\theta(t)} U$ for any $V$ that is d-independent of $U$ iff $U_{(t)} \leq_{\theta(t)} U$ for all $t \geq 0$. 
Proof. With same steps in theorem 8 , and by using (2.4) and (5.1) and we can achieve its proof.

In the following example, we can illustrate Theorem 5.3 and Proposition 5.1.

Example 5.1 : Let $U$, and $V$ denote the random variable have distribution functions $G_{1}$, and $G_{2}$ respectively, which are given by

$$
G_{1}(u)=\left\{\begin{array}{cc}
\frac{u}{b} & \text { for } u \in[0, b) \\
1 & \text { for } u \geq b
\end{array} \text { and } G_{2}(u)=1-\exp (-\lambda u), u \in \mathbb{R}^{+} .\right.
$$

and thus $G_{U_{(V)}}(u)=u \lambda$. It is clear that

$$
\frac{\int_{0}^{u} v G_{U_{(V)}}(v) d v}{G_{U_{(V)}}(u)}=\frac{\int_{0}^{u} v G_{1}(v) d v}{G_{1}(u)}=\int_{0}^{u} v G_{(u)}(v) d v=\frac{u^{2}}{3} .
$$

Hence, $U_{(V)} \leq_{S M P} U \Leftrightarrow U_{(t)} \leq_{S M P} U$. In addition, by using (2.2), we get that $U_{(t)} \varliminf_{\theta(t)} U$, also, $U_{(V)} \nless_{\theta(t)} U$.

\section{References}

[1] R.E. Barlow and F. Proschan, Statistical Theory of Reliability and Life Testing, McArdle Press, Silver Spring, 1981.

[2] I. Dewan and B. Khaledi, On stochastic comparisons of residual life time at random time, Statist. Probab. Lett. 88 C (2014), 73-79

[3] W. J. Hall and J. A. Wellner, Mean Residual Life, in: Statistics and Related Topics, M. Csorgo, J. N. K. Rao and A. K. Md. E. Saleh (eds.), North Holland, Amesterdam, (1981), 169-184.

[4] S. Karlin, Total Positivity, Vol. I., Stanford University Press, Stanford, California, 1968.

[5] M. Kayid and S. Izadkhah, Mean inactivity time function, associated orderings and classes of life distributions, IEEE T. Reliab. 63, 2, (2014), 593-602

[6] X. Li and M. J. Zuo, Stochastic comparison of residual life and inactivity time at a random time, Stoch. Models, 20, 2 (2004), 229-235.

[7] X. Li and Z. Zhang, Some stochastic comparisons of conditional coherent systems, Appl. Stoch. Models Bus. Ind, 24, 6 (2008), 541-549.

[8] M. Mahdy, Characterization and preservations of the variance inactivity time ordering and the increasing variance inactivity time class, Journal of Advanced Research, 3 (2012), 29-34.

[9] M. Misra, N. Gupta, I. Dutt-Dhariyal, Stochastic properties of residual life and inactivity time at a random time, Stoch. Models, 24, 1 (2008), 89-102. 
[10] A.K. Nanda, H. Singh, N. Misra and P. Paul, Reliability properties of reversed residual lifetime, Comm. Statist. Theory Methods, 32, 10 (2003), 2031-2042.

[11] M. Shaked and J. G. Shanthikumar, Stochastic Orders. Springer, New York, 2007.

[12] W. L. Smith, on the cumulants of renewal processes. Biometrika, 46, (1959), 1-29.

[13] Y. Dequan and C. Jinhua, Some results on the residual life at random time, Acta Math. Appl. Sin, 16, 4 (2000), 436-443. 